\title{
EELS Investigations of Carbon-rich Boron Carbide Nanomaterials
}

\author{
Jesse Dampare ${ }^{1}$, Mobolaji Zondode ${ }^{1}$, Sz-Chian Liou ${ }^{2}$, Birol Ozturk ${ }^{1}$, Hongtao $\mathrm{Yu}^{2}$, and Yucheng Lan ${ }^{1}$ \\ 1. Department of Physics and Engineering Physics, Morgan State University, Baltimore, MD. \\ 2. Department of Materials Science and Engineering, University of Maryland, College Park, MD. \\ 3. Department of Chemistry, Morgan State University, Baltimore, MD.
}

Boron carbide has been widely utilized in industry and military because of its low mass-density, superhigh hardness and good electric conductivity [1,2]. It is predicted that its excellent mechanical properties can be enhanced in nanomaterials [3]. The material is also one kind of thermoelectric compound working at high temperatures above $1000{ }^{\circ} \mathrm{C}[4]$. However, its thermoelectric properties, such as Seebeck coefficient and figure-of-merit, are low and need to be enhanced significantly for potential applications. Nanostructuring is an effective approach to improve thermoelectric properties [5] and boron carbide nanocomposites with high performances can be bottom-up-ed from nanomaterials by the approach. So boron carbide nanomaterials play a key role as starting materials of the carbide nanocomposites. Here, carbon-rich boron carbide nanomaterials are synthesized and characterized by electron energy-loss spectroscopy (EELS) on transmission electron microscope (TEM).

The boron carbide nanomaterials were synthesized by a microwave-assistant carbothermic reaction. The synthesized black materials were characterized by X-ray diffraction, as shown in Figure 1. The phase is not boron, carbon, nor $\mathrm{B}_{4} \mathrm{C}$. The UV-vis spectrum (inset in Figure 1) indicated that the band-gap of the nanomaterials is $2.30-2.50 \mathrm{eV}$, larger than that of boron-rich $\mathrm{B}_{\mathrm{x}} \mathrm{C}$ compounds $(2.09 \mathrm{eV})$ [6]. In order to determine the chemical composition of the nanomaterials, EELS technique was utilized here to characterize the nanomaterials. EELS investigations were carried out on a JEOL 2100 FEG TEM equipped with an electron energy loss spectrometer.

EELS spectra and spectrum imaging were collected on one piece of the as-synthesized nanomaterials (Figure 2), which is consisted of nanocrystals. EELS spectra were collected from the marked region and a typical EELS spectrum is shown in Figure 3. Figure 3 indicated that the material is composited of boron and carbon. The ratio of boron to carbon is about 1:2. The high content of carbon maybe come from the un-reacted carbon in the composites or a pure carbon-rich single phase. Here no any diffraction rings of amorphous carbon were observed from selected area electron diffraction patterns (not show here). Therefore, it is reasonable to assume that the synthesized nanomaterials are one kind of carbon-rich $\mathrm{BC}_{2}$ single phase. More work is being carried out to synthesize boron-rich $\mathrm{B}_{\mathrm{x}} \mathrm{C}$ phase. The zoomed spectrum (inset in Figure 3) indicted that the boron K-edge is consisted of a sharp edge and one broad edge, similar as reported [7,8].

In order to characterize the boron distribution in the materials, EELS spectrum imaging was carried out in the marked region shown in Figure 2. A spectrum image is shown in Figure 4a. Based on the spectrum image shown in Figure 4a, a boron EELS mapping was produced and shown in Figure 4b. It is clearly shown that boron uniformly distributed in the whole region. Carbon mapping (not shown here) indicted that carbon uniformly distributed in most regions of the material too. Therefore, the 
synthesized nanomaterials should be uniform $\mathrm{BC}_{2}$ nanomaterials. The detailed crystalline structure of the materials is being carried out [9].

References:

[1] F. Thevenot, J. Euro. Ceram. Soc. 6 (1990) p. 205.

[2] V. Domnich et al, J. Am. Ceram. Soc. 94 (2011) p. 3605.

[3] K. M. Reddy et al, Nat. Commun. 3 (2012) p. 1052.

[4] Z. F. Ren et al, Advanced Thermoelectrics: Materials, Contacts, Devices, and Systems (2017).

(CRC Press).

[5] B. Poudel et al, Science 320 (2008) p. 634.

[6] H. Werheit, J. Phys.: Condens. Matter 18 (2006) p. 10655.

[7] Y. G. Lu et al, Carbon, 86 (2015) p. 156.

[8] R. Z. Ma et al, Chem. Phys. Lett. 364 (2002) p. 314.

[9] The authors acknowledge the support from ESI/JHU0-MEDE Program.

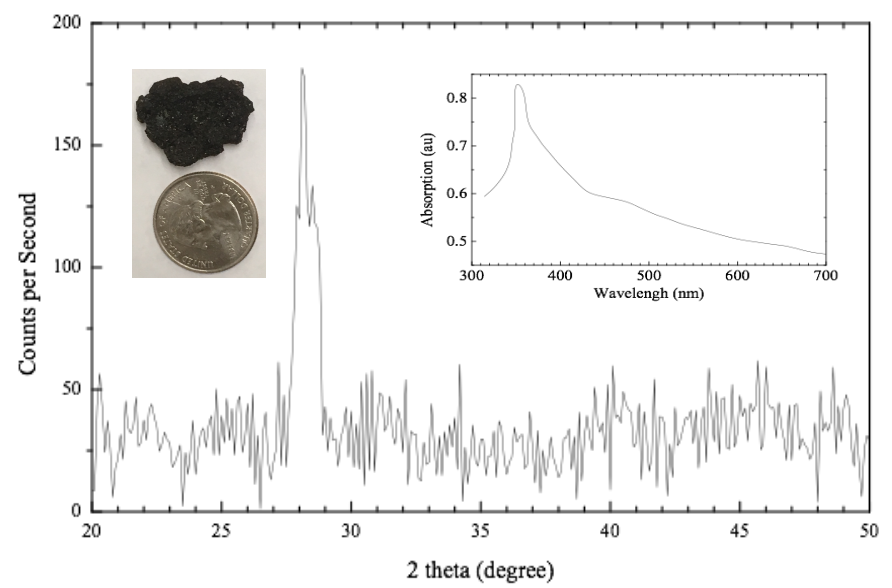

Figure 1: An X-ray diffraction pattern of $\mathrm{BC}_{2}$ nanocomposites. Left inset: optical image of assynthesized nanocomposites. Right inset: UV-vis

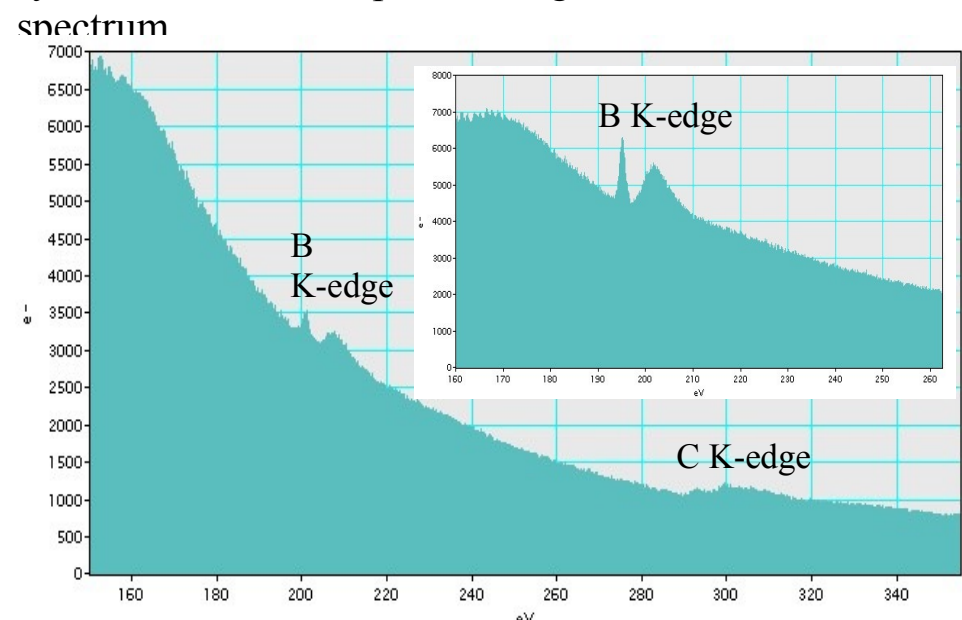

Figure 3: An EELS spectrum showing boron and carbon edges. Inset: zoomed boron K-edge.

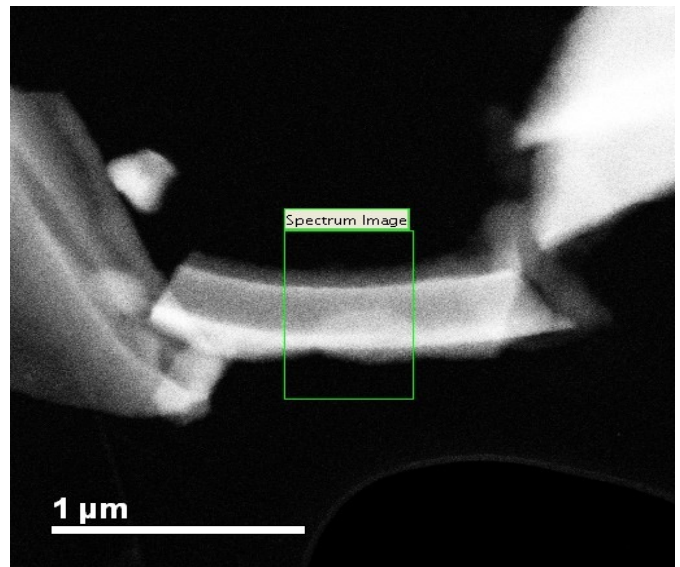

Figure 2: An STEM image of $\mathrm{BC}_{2}$ nanocomposites. The spectrum image region is marked.
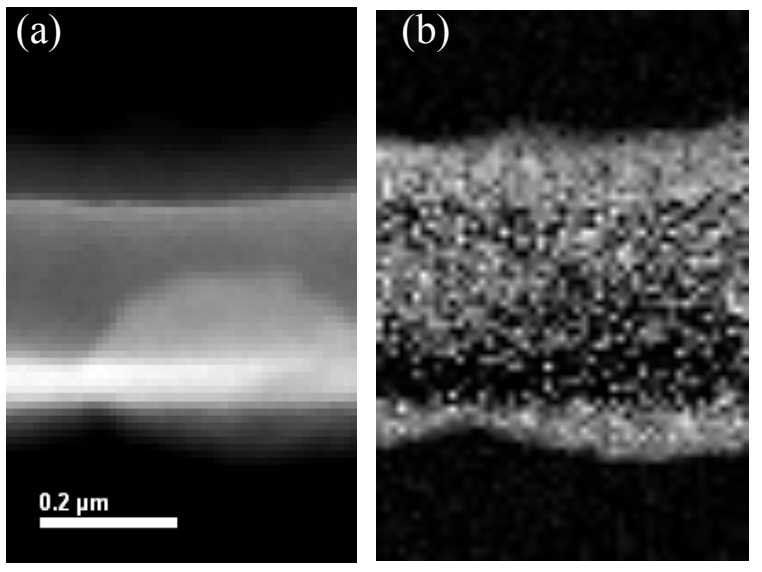

Figure 4: (a) EELS spectrum image. (b) Boron EELS mapping image. 